\title{
Improved frequency table's measures of skewness and kurtosis with application to weather data
}

\begin{abstract}
The statistic" Midpoint" used as a magnitude of observations in each class of a frequency table leads to biased estimates of the two measures of shape of a distribution, skewness, and kurtosis. This research proposed three new statistics, the mean, median, and midrange of observations in each class instead of the midpoint. Simulation results using samples from normal, uniform, exponential distributions, and the real Istanbul weather data indicated that measures that used the mean as a representative of observations in each class outperformed the other measures of skewness and kurtosis.
\end{abstract}

Keyword: Frequency table; Skewness; Kurtosis; Midpoint; Number of classes 